Running Head: EMOTION REPAIR AND THE DIRECTION OF ATTENTION

\title{
Emotion Repair and the Direction of Attention in Aversive Contexts: Evidence from an Attention-Demanding Task
}

\author{
Adam K. Fetterman, Konrad Bresin, and Michael D. Robinson \\ North Dakota State University
}

Note: Correspondence can be directed to Adam Fetterman, Psychology Department, North Dakota State University (NDSU Dept. 2765), PO Box 6050, Fargo, ND 58108-6050 (Email: Adam.Fetterman@ndsu.edu; Phone: 701-231-5398). The authors acknowledge support from NSF (BCS 0843982). 


\begin{abstract}
Two studies (total $N=145$ ) examined the novel idea that individual differences in emotion repair may relate to the attention deployment stage of emotion regulation. More specifically, it was hypothesized that high repair individuals would be able to maintain focus on an attentiondemanding task in an aversive context, but that low repair individuals would not, in both cases relative to a control condition. This sort of interactive hypothesis was supported in Study 1, which manipulated aversive events through the use of concurrent auditory stimulation and conceptual replication was found in Study 2. Together, the two studies offer suggestive evidence for the role of attention direction in emotion repair.
\end{abstract}

KEYWORDS: Emotion Repair, Emotion Regulation, Attention Direction, Performance 


\section{Emotion Repair and the Direction of Attention in Aversive Contexts: Evidence from an Attention-Demanding Task}

Some individuals may spontaneously self-regulate their reactions to aversive events. Such considerations gave rise to the Trait Meta-Mood Scale (TMMS: Salovey, Mayer, Goldman, Turvey, \& Palfai, 1995). Of particular importance from an emotion regulation perspective, the scale's authors created and validated an individual difference measure of emotion repair. This measure negatively predicted depression, positively predicted subjective well-being, and was linked to more pleasant emotional states several minutes following a negative emotional manipulation. Similar results have been reported by others (e.g., Hemenover, 2003).

Individual differences in emotion repair appear pronounced, but how do they operate? The authors of the emotion repair scale (Salovey et al., 1995) did not advance a particular mechanism for emotion repair, but it seems possible that attentional factors might be involved. Consider one of the items of the scale: "When I become upset I remind myself of all the pleasures in life." This statement may implicate the attention deployment stage of emotion regulation (Gross \& Thompson, 2007), whereby some individuals may be better able to selfregulate their reactions to aversive events by distracting themselves from such events. In numerous experimental studies, Mischel and colleagues have shown that distraction manipulations of attention do in fact reduce emotional reactivity (Metcalfe \& Mischel, 1999).

Might people high in emotion repair distract themselves from aversive events? We do not know the answer to this question, but it is plausible that they might do so. A potential way of examining whether this is the case is to manipulate aversive events in combination with an attention-demanding task (Jostmann \& Koole, 2007). Those who cannot distract themselves from 
aversive events, in tasks like this, will exhibit difficulties in the context of such events. Fine motor control is particularly attention-demanding in that it requires constant adjustments to perform such tasks accurately (Slifkin \& Newell, 1998). We therefore used such a task in the present studies.

In both studies, we hypothesized interactions between individual differences in emotion repair and context (aversive versus control) in predicting fine motor control. The nature of the interactions should be as follows. Due to their hypothesized focus on aversive events, low repair individuals should exhibit worse control in aversive relative to neutral contexts. By contrast, high repair individuals should exhibit better control in aversive relative to neutral contexts. Interactive results of this type would contribute to our understanding of individual differences in emotion repair and how attentional factors may be relevant in understanding them.

Study 1

Study 1 involved playing neutral or aversive sound clips (Bradley \& Lang, 1999) over headphones. We hypothesized that the sound clip manipulation would interact with individual differences in emotion repair (Salovey et al., 1995) to predict fine motor control, an especially attention-demanding task (Slifkin \& Newell, 1998).

\section{Method}

\section{Participants and General Procedures}

Participants were 78 (40 female; $M$ age $=20.00 ; 87 \%$ Caucasian) undergraduates from North Dakota State University who received course credit. Groups were run in sizes of 6 or less and each participant was seated at his/her own private computer.

\section{Emotion Repair}


We administered the six-item emotion repair scale from the TMMS (Salovey et al., 1995). Participants were asked to indicate the extent to which $(1=$ strongly disagree; $5=$ strongly agree) they typically engage in emotion repair efforts when upset (e.g., "I try to think good thoughts no matter how badly I feel"). Individual differences in emotion repair, as assessed by this scale, positively predict beliefs that one can regulate negative mood states (Salovey et al, 1995) and strongly correlate with other trait scales purported to assess similar emotion regulation tendencies (Petrides, Perez-Gonzalez, \& Furnham, 2007). Alpha was .65 $(M=3.78, S D=.58)$.

\section{Attention-Demanding Task}

Instructions. The computer program informed participants that we were interested in their ability to do two different tasks at the same time. They were to listen to sound clips presented over headphones for a vaguely described subsequent memory test, which was not actually administered. While doing so, they were to move a Saitek Aviator-01 Dual Throttle joystick cursor to a presented visual target on the computer screen and hold it as steady as possible on the presented visual target for the remainder of the trial. In both studies, E-Prime programming software (Schneider, Eschman, \& Zuccolotto, 2001) was used.

Aversive Event Manipulation. Sound clips were selected from the IAD-2 database of Bradley and Lang (1999), all of which were almost exactly 6 seconds in duration. On the basis of such norms, fifteen neutral sound clips (e.g., cattle sounds, yawning) were chosen, as were 15 aversive (e.g., screaming, vomiting) sound clips. By the Bradley and Lang norms, the aversive sounds were more unpleasant $(M=2.24$ along a $1-9$ valence scale $)$ than the neutral sounds $(M=$ 4.84). They were also higher in arousal ( $M \mathrm{~s}=7.06$ versus 4.68 along a $1-9$ arousal scale).

Trial Procedures. A visual target (a white + sign) was presented in a random location on the computer screen. A joystick cursor (also a white + sign) was then presented at center screen 
and participants were to move the joystick cursor to the visual target, which then turned yellow and remained stationary. Immediately thereafter, a sound clip was presented over headphones. Participants were to hold the joystick cursor as steady as possible on the presented target for the next $6000 \mathrm{~ms}$. Within this window, joystick position was sampled every $50 \mathrm{~ms}$, thus resulting in 120 samples per trial. Each sample recorded the extent to which the current joystick position deviated from that of the visual target, in pixel based terms, in both $\mathrm{x}$ - and $\mathrm{y}$-axis coordinates. After the $6000 \mathrm{~ms}$, participants were instructed to return to center screen. Each clip was played twice, meaning that there were 30 aversive trials and 30 neutral trials, a manipulation of a withinsubject type. Clips were assigned to trial number at random.

Performance. Participants were necessarily quite close to the target at the start of the trial (i.e., they had to be within 5 pixels of it) and the sound clips convey their meaning over time. Thus, performance in the attention-demanding task was quantified in terms of exhibited difficulties over time. For every joystick sample, we first calculated the Euclidean distance between the joystick position and the visual target using the Pythagorean Theorem. We then correlated the trial-specific sample number (from 1 to 120) with such Euclidean distance scores. Higher indices thus reflect losses in fine motor control with time. Such trial-specific values were then averaged, separately so, by emotion condition and participant-level emotion repair. Owing to the within-subject nature of the manipulation, each participant had one performance score for the aversive condition and a second performance score for the neutral condition.

\section{Results and Discussion}

Control loss scores were examined in a General Linear Model (GLM) analysis as a function of emotion condition (neutral versus aversive) and continuous, though z-scored, variations in emotion repair (Robinson, 2007). Main effects were not significant, $F$ s $<1$, but the 
hypothesized interaction was significant, $F(1,76)=4.50, p<.05$, partial eta square $=.06$. Estimated means by condition at prototypically low (-1 SD) versus high $(+1 S D)$ levels of emotion repair are displayed in Figure 1 (Aiken \& West, 1991). Relative to the control condition, aversive sounds engendered losses of control at low levels of emotion repair, but gains of control at high levels of emotion repair. As performance in the task necessitated considerable attention (Slifkin \& Newell, 1998), the results indicate that high (but not low) repair people maintain focus and attention to the task at hand during aversive events, relative to a control condition.

Study 2

The closer in time one is to a future aversive event, the more emotionally arousing it becomes (Bechara, 2004; Monat, Averill, \& Lazarus, 1972). Accordingly, we could induce an aversive event in Study 2 by telling individuals, accurately so, that they would receive a white noise blast, versus not, upon completion of the trial. An interaction parallel to Study 1, in the context of a very different manipulation of aversive experiences, was hypothesized.

\section{Method}

Participants, General Procedures, and Personality Assessment

Sixty-seven (37 female; $M$ age $=19.06 ; 86 \%$ Caucasian) undergraduates from North Dakota State University received course credit. General procedures were identical to Study 1 and emotion repair was again assessed by the relevant TMMS scale (alpha $=.78 ; M=3.70 ; S D=$ $.72)$.

\section{Attention-Demanding Task}

Instructions. Instructions were identical to Study 1 except that it was stated that half of the trials would be followed by a noise blast and half would be followed by silence. 
Aversive Event Manipulation. In the aversive condition, we presented $80 \mathrm{~dB}$ white noise blasts over headphones for $4500 \mathrm{~ms}$ immediately after the motor control portion of the trial was done, whereas no sound was presented over headphones in the control condition. Previous studies confirm the aversive nature of this manipulation (e.g., Fetterman, Robinson, Gordon, \& Elliot, 2011). The manipulation was anticipatory in nature, however, as detailed next.

Trial Procedures. Participants first received a $2000 \mathrm{~ms}$ message informing them that the trial was one on which they would receive a noise blast over headphones after each trial versus not. Subsequent to the $2000 \mathrm{~ms}$ message, the motor control portion of the trial began. Its procedures were identical to Study 1 except that the time frame was shorter (2000 ms). As joystick position was sampled every $50 \mathrm{~ms}$, there were 40 samples per trial. There were 30 trials for each of the two conditions: aversive versus control, a within-subject design. Noise trials were followed by noise and control trials were followed by silence, exactly as indicated initially.

Performance. Control loss scores were computed in a manner parallel to Study 1 and then averaged for each condition and each participant's emotion repair score for analysis purposes.

\section{Results and Discussion}

A GLM analyzed control loss scores as a function of condition and z-scored individual differences in emotion repair (Robinson, 2007). The two main effects were not significant, $p$ s > .15 , but there was a significant interaction, $F(1,65)=6.06, p<.05$, partial eta square $=.09$. Estimated means were calculated as in Study 1 and they are graphically displayed in Figure 2. Again, fine motor control was better in the aversive condition relative to the control condition at a prototypically high $(+1 S D)$ level of emotion repair, but was worse at a prototypically low (-1 $S D)$ level of emotion repair. Study 2 thus conceptually replicates Study 1 in revealing that high 
(but not low) emotion repair individuals maintain task performance during aversive events, potentially as a way to self-regulate their reactions to them.

\section{General Discussion}

The emotion repair scale administered was created to assess individual differences in the extent to which individuals mitigate their negative emotional states, but is not otherwise committed to a particular view of how they do so (Salovey et al., 1995). In this sense, it is an admirably general scale that would seem to have wide utility. This admirable quality aside, the emotion regulation literature has increasingly sought to understand how it is that some people are better able to self-regulate their reactions to aversive events or experiences.

On the basis of previous theorizing (e.g., Metcalfe \& Mischel, 1999), we thought that the deployment of attention might be relevant in understanding individual differences in emotion repair. More specifically, it is possible that high repair individuals distract themselves from aversive events. If they do so, and are asked to perform an attention-demanding task, they should perform that task reasonably well in an aversive context relative to a control context. By contrast, low repair individuals might dwell upon aversive events, resulting in poorer performance in this same aversive context. If such ideas are valid, there should be an interaction between emotion condition (aversive versus control) and individual differences in emotion repair. We obtained interactions of this type in two studies in which aversive events co-occurred with the performance task (Study 1) or were anticipated at the end of each trial (Study 2). Accordingly, we suggest that individual differences in emotion repair might be understood in terms of the attention deployment mechanism of emotion regulation highlighted by Gross and Thompson (2007), a mechanism arguably understudied in the personality realm. 
Salovey et al. (1995) reported some evidence for the idea that higher levels of emotion repair predicted lesser levels of negative emotion to a negative event manipulation 10s of minutes later. Our results encourage the idea that individual differences in emotion repair are evident in more immediate reactions to aversive events as well. In this sense, our results are consistent with an emerging body of work favoring the idea of automatic emotion regulation processes (Mauss, Bunge, \& Gross, 2007). It remains to be determined how the fast-acting dynamics found in our studies relate to the sorts of emotion-related changes reported by Salovey et al. (1995) and Hemenover (2003).

There are several limitations to the findings as well as future directions of research that should be pursued. It may be of value to conceptually replicate the present interactive findings using another attention-demanding task, such as a working memory task (Schmeichel, Volokhov, \& Demaree, 2008). This is a particularly useful direction because our findings may reflect motor control processes rather than attentional processes narrowly considered. The aversive events were aversive, but not particularly ego-threatening. It would therefore be of value to examine similar dynamics following aversive events of a more ego-threatening type (e.g., poor performance in a purported ability task). We examined fast-acting regulatory processes, but other designs - especially of a between-subjects nature - are warranted to examine longer-lagged consequences of individual differences in emotion repair. Nonetheless, modifications of our task procedures would seem to possess wider utility in understanding the role of attention in relation to other sorts of cues or manipulations. For example, problem drinkers may display control losses following pictures of alcoholic drinks relative to pictures of non-alcoholic drinks. Other individual differences - such as rumination and self-control - would similarly seem to lend themselves to an analysis of the present type. 


\section{References}

Aiken, L., \& West, S. (1991). Multiple regression: Testing and interpreting interactions. Thousand Oaks, CA: Sage Publications, Inc.

Bechara, A. (2004). A neural view of the regulation of complex cognitive functions by emotion. In: P. Philippot, R. S. Feldman, P. Philippot, R. S. Feldman (Eds.), The regulation of emotion (pp. 3-32). Mahwah, NJ: Lawrence Erlbaum Associates Publishers.

Bradley, M. M., \& Lang, P. J. (1999). International affective digitized sounds (IADS): Stimuli, instruction manual and affective ratings (Tech. Rep. No. B-2). Gainesville, FL: The Center for Research in Psychophysiology, University of Florida.

Fetterman, A. K., Robinson, M. D., Gordon, R. D., \& Elliot, A. J. (2011). Anger as seeing red: Perceptual sources of evidence. Social Psychological and Personality Science, 2, 311316.

Gross, J. J., \& Thompson, R. A. (2007). Emotion regulation: Conceptual foundations. In: J. J. Gross (Ed.), Handbook of emotion regulation (pp. 3-24). New York: Guilford Press.

Hemenover, S. H. (2003). Individual differences in rate of affect change: Studies in affective chronometry. Journal of Personality and Social Psychology, 85, 121-131.

Jostmann, N. B., \& Koole, S. L. (2007). On the regulation of cognitive control: Action orientation moderates the impact of high demands in Stroop interference tasks. Journal of Experimental Psychology: General, 136, 593-609.

Mauss, I. B., Bunge, S. A., \& Gross, J. J. (2007). Automatic emotion regulation. Social and Personality Psychology Compass, 1, 146-167.

Metcalfe, J., \& Mischel, W. (1999). A hot/cool-system analysis of delay of gratification: Dynamics of willpower. Psychological Review, 106, 3-19. 
Monat, A., Averill, J. R., \& Lazarus, R. S. (1972). Anticipatory stress and coping reactions under various conditions of uncertainty. Journal of Personality and Social Psychology, 24, 237253.

Petrides, K. V., Pérez-González, J., \& Furnham, A. (2007). On the criterion and incremental validity of trait emotional intelligence. Cognition and Emotion, 21, 26-55.

Robinson, M. D. (2007). Lives lived in milliseconds: Using cognitive methods in personality research. In: R. W. Robins, R. C. Fraley, \& R. Krueger (eds.), Handbook of research methods in personality psychology (pp. 345-359). New York: Guilford Press.

Salovey, P., Mayer, J. D., Goldman, S., Turvey, C., \& Palfai, T. P. (1995). Emotional attention, clarity, and repair: Exploring emotional intelligence using the Trait Meta-Mood Scale. In: J. W. Pennebaker (Eds.), Emotion, disclosure, and health (pp. 125-154). Washington, DC: American Psychological Association.

Schmeichel, B. J., Volokhov, R. N., \& Demaree, H. A. (2008). Working memory capacity and the self-regulation of emotional expression and experience. Journal of Personality and Social Psychology, 95, 1526-1540.

Schneider, W., Eschman, A., \& Zuccolotto, A. (2001). E-Prime user's guide. Pittsburg, PA: Psychology Software Tools, Inc.

Slifkin, A. B., \& Newell, K. M. (1998). Is variability in human performance a reflection of system noise? Current Directions in Psychological Science, 7, 170-177. 
Figure 1

Motor Control Loss by Emotion Condition and Emotion Repair, Study 1

Figure 2

Motor Control Loss by Emotion Condition and Emotion Repair, Study 2 